\title{
Magnetic Transducers in Biosensors and Bioassays
}

\author{
K. Larsson ${ }^{1}$, K. Kriz ${ }^{1,2}$, D. Kriz*1,2 \\ ${ }^{I}$ European Institute of Science, Research Park IDEON, SE-223 70 Lund, Sweden \\ ${ }^{2}$ Department of Pure and Applied Biochemistry, Chemical Center, University of Lund, P.O. Box 124, SE-221 00 Lund, Sweden
}

In affinity biosensor technology, antibodies are employed as the recognition element in close conjunction with a transducer. Much work has been put into developing effective transducers, and a number of technologies exist.

In this review various works that we have found significant to the field of magnetic transducers in bioanalysis have been critically studied in order to describe the status and development of magnetic measurements in biosensors and bioassays. Our group has previously suggested that magnetic transducers in combination with magnetically labelled antibodies may be used in this context and this so-called magneto immunoassay (MIA) technique will be dealt with in some detail.

\section{Introduction}

Antibodies are made by all animals as an immunological response to the presence of a foreign invader in the blood. The inherent specificity of an antibody is directed towards the epitope of the antigen capable of eliciting the immunological response. Due to their high specificity, which is an essential factor in determining drugs, metabolites, hormones or proteins in the body accurately, antibodies $[1,2]$ as well as enzymes [3] have been employed as analytical tools in biosensor and bioassay technology. Enzymes are much simpler to utilise due to the fact that they, in addition to binding, also have the ability to convert a molecule. This ability is very useful as it might give rise to suitable physical changes, which can be detected employing various transducers [4]. However, the selection of analytes becomes rather limited when using enzymes. Here antibodies provide the basis for a large selection of potential analytes including proteins and cells. The challenge when using antibodies lies in the development of various labelling techniques and transducers to detect the binding of the analyte $[2,5,6,7,8,9]$. Both in bioassay technology and biosensor technology various strategies have been utilised.

Since the molecular interaction between an antibody and an antigen does not result in any analyte conversion, typically either of these counterparts have been coupled to enzyme markers which have catalytic properties that can be monitored optically [10], electrochemically [2] or thermometrically $[11,12]$. Other common labelling techniques found in bioassays employ the use of radioactive compounds, fluorophores and luminescence markers. Radioimmunoassays (RIA), established in 1959 [13], use radioactive markers and are considered today as one of the most important techniques used in the clinical and biochemical fields for the quantitative analysis of hormones, steroids and drugs due to the relatively high sensitivities (1$500 \mathrm{pM})$ these markers can offer. Although large centralised

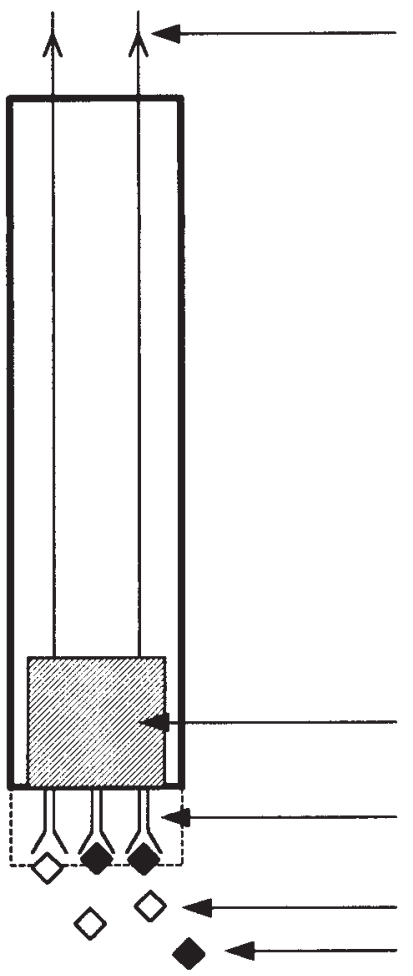

\section{Electrical output signal}

\section{Coil - magnetic transducer \\ Immuno - layer \\ Other substances \\ Analyte (labelled)}

Figure 1. The conceptual arrangement of a magneto biosensor based on a magnetic transducer. The analyte is bound to an immuno-layer consisting of immobilised antibodies. Upon binding the closely positioned magnetic transducer (coil) detects local changes in magnetic permeability, as an increase of the superparamagnetically labelled analyte is concentrated at the immuno-layer. 
laboratories use the already established radioimmunoassays since highly automated equipment is available for detection, smaller laboratories and hospital clinics are switching over to the enzyme-linked immunosorbent assays (ELISA) even though lower sensitivities are obtained when using these assays. ELISA does not require the use of high-cost equipment and the enzyme markers do not present the radiological hazards normally associated with RIA, factors which make this method suitable for smaller laboratories [14]. The use of fluorophores and luminescence markers has also been employed in immunoassays although to a somewhat lesser extent than their radioactive and enzyme counterparts. These markers offer sensitivities normally associated with radioactive markers, but possess similar advantages analogous to the enzyme markers [15]. For a review on clinical applications of immunoassays see Anderson et al., 1997 [16].

In biosensor technology, antibodies have been employed as the recognition element in close conjunction with a transducer, giving rise to the category of noncatalytic biosensors, referred to as affinity sensors. The binding of larger analytes such as proteins or cells causes mass enrichment on the transducer surface which may cause its resonance frequency to change, more commonly known as piezo effect [17, 18], or locally change the refractive index, also known as surface plasmon effect $[19,20]$. Others involve the enrichment of fluorescent or light absorbing substances [21]. Our group suggested in 1995 that magnetic transducers might also be used in this context [22]. Figure 1 illustrates the conceptual arrangement of such a magneto biosensor.

\section{Paramagnetism}

Superparamagnetic materials are strongly affected when exposed to external magnetic fields. They contain small clusters of atoms, so called domains, which always have all of their magnetic dipoles oriented in the same direction. This spontaneous orientation can only be explained by quantum mechanics. The size of a domain is typically $1-10 \mu \mathrm{m}$. In paramagnetic materials the overall magnetic dipole of many domains is oriented in the direction toward an external magnetic field and enhances it. Superparamagnetic materials, which normally contain metals such as iron, nickel or cobalt, exhibit a much higher ratio of domains that orient in the direction of the external magnetic field. In order to quantify a materials ability to interact with external magnetic fields, the terms magnetic permeability and magnetic susceptibility have been introduced. The relative magnetic permeability, $\mu_{r}$, is a measure of a materials ability, compared to vacuum, to contribute to a magnetic field. The majority of materials such as water, many metals, biological materials and gases have $\mu_{\mathrm{r}}$ equal to 1 . Superparamagnetic materials such as the iron oxide magnetite, $\mathrm{Fe}_{3} \mathrm{O}_{4}$, has $\mu_{\mathrm{r}}$ equal to 600-1000 000 . The magnetic susceptibility, $\chi_{\mathrm{m}}$, is defined as $\chi_{\mathrm{m}}=\mu_{\mathrm{r}}-1$, and is frequently used due to historical reasons.

Once the external magnetic field has been removed the overall magnetic dipoles of the domains become randomly oriented again. Remanence is a measure on how long the field orientation of the domains is retained. In permanent magnetic materials this field orientation is kept indefinitely.

When preparing small superparamagnetic particles, size becomes an important factor. As mentioned, the dimension of domains is typically $1-10 \mu \mathrm{m}$, and in order to achieve permanent magnetic properties the clusters of domains must be organised in larger crystals. For bioanalytical applications the desired size distribution lies in the range 10-300 nm. Such superparamagnetic bioanalytical particles show low remanence and can thus, exist in stable colloidal form even after having been exposed to an external magnetic field.

\section{Paramagnetic fluids as labels}

Paramagnetic fluids contain nanosized superparamagnetic particles. These particles have a superparamagnetic centre, such as magnetite, which has been coated with some type of polymer layer such as dextran, polyethyleneglycol, protein, or a fatty acid bilayer [23]. Until now the most common uses for large superparamagnetic particles in biotechnology has been for the labelling and separation of cells and macromolecules. These particles have a size of several micrometers $[24,25]$. Today there are a number of established companies, such as Dynal and PerSeptive, which commercially offer this type of particles for separation purposes. Large superparamagnetic particles for separation were already used by several groups in solid-phase radioimmunoassays by the mid 1970's, where antibodies were covalently linked to the magnetic particles in order to carry out the subsequent separation between the antibody-bound and free fractions [26, 27]. However, it was not until recently that it was demonstrated that nanosized, dextran coated superparamagnetic particles could be used for bioanalytical applications [28, 29].

The use of superparamagnetic particles as markers on antibodies has several advantages over existing markers in immunoassays. They are completely non-hazardous both to people and the environment, which is a benefit in handling and waste treatment, and the risk of interference from other components in the sample is very small, as the vast majority of compounds are not paramagnetic. Furthermore, the magnetic particles are relatively cheap, and have essentially unlimited shelf life.

\section{Biomagnetic transducers}

\section{Induced current transducers}

The magnetic stimulation and biomagnetic detection of an action current in nerve tissue has been investigated using an external magnetic field together with crustacean nervous tissue as the biological transducing element [30]. An action current was induced by a high-frequency magnetic pulse from a wire-wound toroid that encircles the nerve fibre and a second toroid measured the response current downfield. This technique has been used to detect and to determine dose-response curves for local anaesthetics that block passage of the action current down the nerve fibre. 
This approach could potentially be used in a biosensor set-up where progression of nerve pulses is effected when the nerve tissue is exposed to different substances. However, this semi-biomagnetic transducer will have the inherit disadvantages of the nongeneral nature of the approach, the complex response signal obtained due to the large number of stimulating substances which affect the nerve, and the interference from electronic noise in the surroundings.

\section{Atomic force microscope based transducers}

One group that has investigated the use of superparamagnetic particles as labels in combination with different magnetic transducers is the US Naval Research Laboratory. They have conceived the Force Amplified Biological Sensor (FABS) which is a biosensor based on magnetic determination and one that employs a solid-phase immunoassay [31]. The transducer employed in the FABS device is a cantilever-beam force transducer, developed originally for atomic force microscopy (AFM), where antibodies directed towards a specific analyte can be coupled to the surface of the cantilever. The operational principle of the FABS device replicates AFM, but uses superparamagnetic particles to exert a force on intermolecular bonds instead of a piezoceramic translator [32]. In this instrument the sample is first introduced onto the transducer followed by washing and the subsequent introduction of the superparamagnetic label which binds specifically to the analyte via antibody-antigen interactions. An electromagnet generates a field that pulls on the superparamagnetic particles and the attached particles exert a force on the cantilever which is directly proportional to the amount of labels attached to the transducer surface and thus, the number of particles bound indicates the concentration of analyte in the sample. Although a portable prototype has been developed, the hardware and chemistry have not been integrated together at the present time.

\section{Magneto resistive transducers}

Recently, the US Naval group announced the underlying principles of their Bead Array Counter (BARC) which is a microfabricated magnetoresistive transducer which can indicate whether or not the particles are removed when pulled by magnetic forces. They postulate by adapting magnetoresistive computer memory technology, it may be possible to fabricate millions of transducers on one chip and detect or screen thousands of analytes, which would be useful in DNA hybridisation assays [33]. Both the FABS and the BARC techniques require the use of rather large superparamagnetic particles $(2 \mu \mathrm{m})$.

\section{Magnetic remanence transducers}

In the magnetic remanence immunoassay Kötitz and coworkers employ nanosized superparamagnetic particles as labels to antibodies and the quantitative determination of the antibody-antigen interaction is based on a magnetic particle relaxation measurement [29]. The operating principle of this technique is based on the fact that the rotational diffusion of the label is hindered by the specific interaction between the antibody and the antigen, which is attached to a solid surface. As a result, the internal reorientation of the magnetic moment of the superparamagnetic particle is affected and the remanence is measured with a dc-SQUID (Superconducting QUantum Interference Device). The feasibility of the instrument has been demonstrated on the detection and determination of collagen type III. Unlike the FABS device, the SQUID measurements require no additional washing steps for the separation of bound and unbound components due to the fact that the signal obtained is specific for only the bound particles since the system measures the remanent sample magnetisation in the absence of an external field. However, the SQUID system requires sophisticated measuring equipment and currently, does not offer portable instrumentation possibilities.

\section{Inductance transducers}

Maxwell bridge based inductance transducers for bioanalysis, illustrated in figure 2 , were originally developed by our

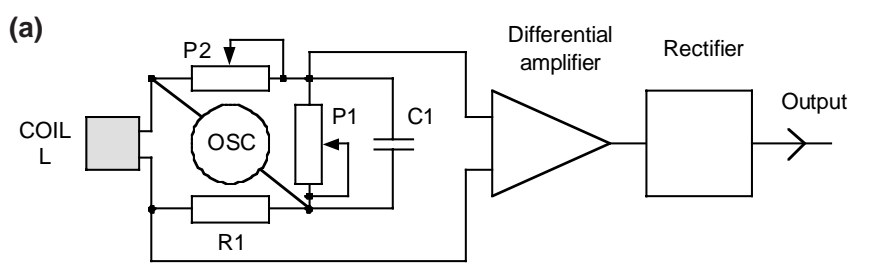

(b)

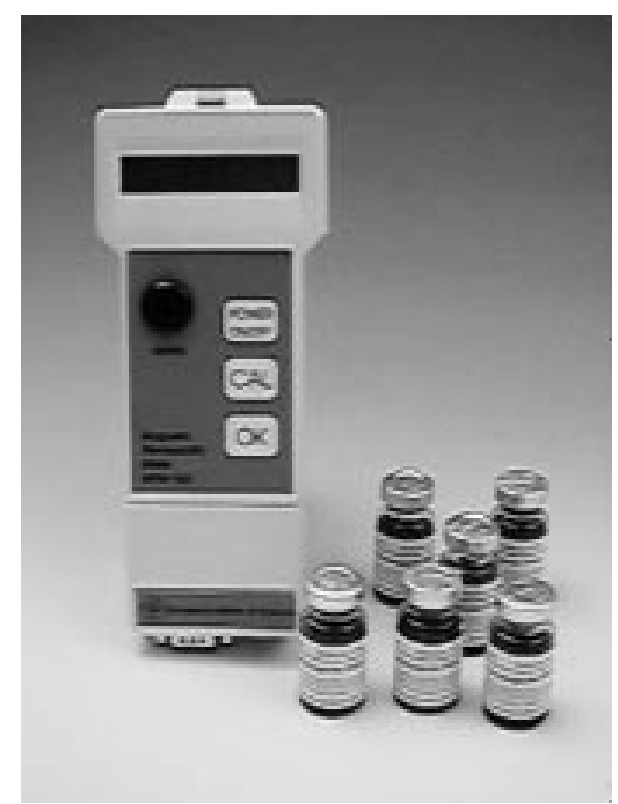

Figure 2 (a) The schematic set-up of a magneto transducer for the determination of magnetic permeability of samples that are introduced into the coil (L). An oscillator providing a sinusoidal wave of $200 \mathrm{kHz}$ is connected to the Maxwell bridge illustrated in the figure. Any changes of the magnetic permeability of the material in the coil gives an inductance change which yields a signal that is processed by the differential amplifier and an amplifier. (b) A microprocessor controlled portable device, developed at the European Institute of Science, intended for detection of relative magnetic permeability. 
group [28]. Such transducers enable the measurement of the relative magnetic permeability of paramagnetic samples (gases, liquids or solids) that are positioned inside a measuring coil. Paramagnetic materials inside a coil influence the inductance of the coil. It is thereby possible to detect changes in relative magnetic permeability by measuring the change in inductance. Different electrical approaches may be utilised for the inductance determination of a coil, for example:

- Placing the coil in an electrical measuring bridge such as the Maxwell bridge [28].

- Measurement of the resonance frequency of a LC-circuit containing the coil [34].

- Measurement of the coil's impedance.

- Dynamic measurement of the current response after having applied a potential pulse to the coil.

- Monitoring the potential response of the coil after applying a current pulse or a non-constant current where the current change per time (di/dt) is kept constant.

- Telemetric measurement based on inductive coupling between two coils.

\section{Magneto immunoassay}

In the magneto immunoassay (MIA) technique an inductance transducer, as described above, is employed to measure the relative magnetic permeability of superparamagnetic markers.

Figure 3 illustrates the working principle of the MIA technology. The reaction is initiated by introducing the sample into a reaction vial containing a mixture of superparamag-

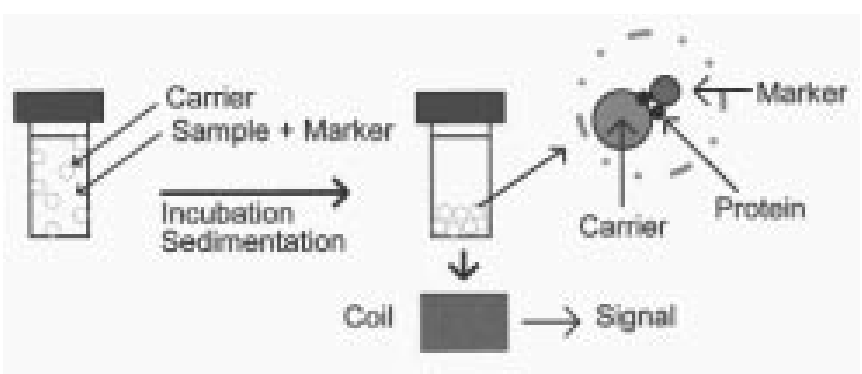

Figure 3. The operational principle of the magneto immunoassay technology. The sample vial contains the functionalised silica carrier, antibody conjugated superparamagnetic marker and the sample to be analysed. The analyte which is present is entrapped in a "sandwich" complex between the carrier and the superparamagnetic particle and subsequently, this complex sediments. The magnetic permeability of the sedimented superparamagnetic markers are determined using an inductance transducer. netically labelled antibodies and carrier particles, which are functionalised silica particles. Subsequently, a "sandwich" complex is formed, where the analyte becomes bound between the superparamagnetic particle and the silica carrier via antibody-antigen interactions. The silica carriers have a larger size $(15-40 \mu \mathrm{m})$ and density, which allows the complex to sediment during a 20 to 30 minutes incubation. If the target analyte is present in the sample, an enrichment of superparamagnetic particles in the sediment will occur as a result of the antibody-antigen recognition. When the sample vial is placed inside the coil the relative magnetic permeability of the superparamagnetic particles enriched in the sediment is determined, which is directly proportional to the concentration of the analyte present in the sample.

Apart from the "sandwich" mode of detection other approaches for the detection of an analyte in solution can be taken. In the rare occasions where the analyte itself is superparamagnetic it can be directly detected by binding to the carrier-coupled recognition element. Another approach is to use a superparamagnetic competitor which has similar affinity to the recognition element as the analyte, and determine the decrease in magnetic permeability with increased analyte concentration.

The superparamagnetic particles currently used in the MIA technology consists of a magnetite core surrounded by a dextran layer. The particles have a size range of 100 to $300 \mathrm{~nm}$. The magnetic interactions and van der Waals attractive forces are overcome by the entropy repulsion energy obtained by the dextran coating and thus, the particles are present as a colloidal solution. The outer dextran shell also enables easy coupling of antibodies or proteins to the exposed hydroxyl groups on the surface of the particles.

We have shown the feasibility of the MIA technology by using Concanavalin A (Con A) as the target analyte [28, 35]. Con $\mathrm{A}$ is a lectin with a high affinity for various sugars, including dextran and glucose. As it complexes with these polysaccharides, it is often used as a model system for immunological systems. When Con A is incubated with the silica carrier particles and the superparamagnetic particles it becomes entrapped between the carrier and the marker via nonselective protein adsorption and affinity binding [35]. The complexes are allowed to sediment, and subsequently, the magnetic permeability of the sediment is measured using the magnetic permeability meter outlined in figure 2 . Results from this study are shown in figure 4.

A detection limit of $250 \mathrm{nM}$ was obtained with this model system. However, by employing smaller silica particles and thereby increasing the surface area for protein attachment, or by improving the superparamagnetic particles and thereby increasing the magnetisation, the sensitivity of the method can be pushed down to the picomolar range.

The MIA technology has the advantages of being easy to perform, fast and robust, which make it particularly suited for in-field and for point-of-care usage. 
a)

Relative Magnetic Permeability (mV)

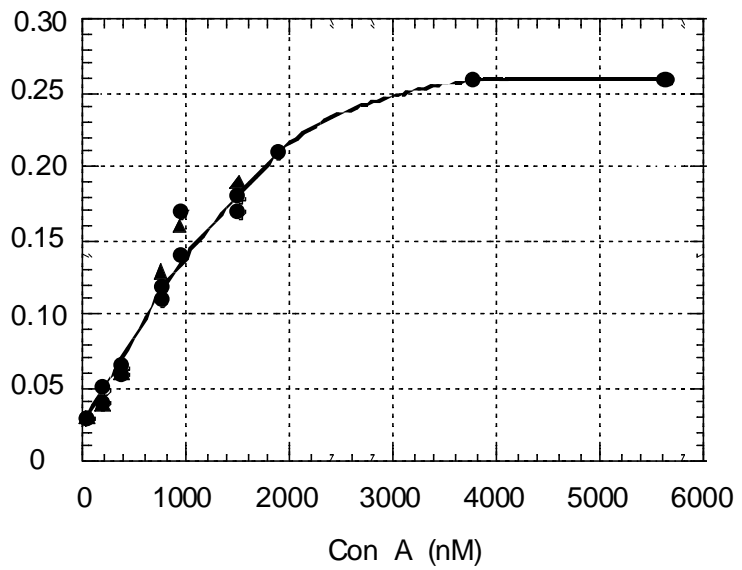

b) Relative Magnetic Permeability (mV)

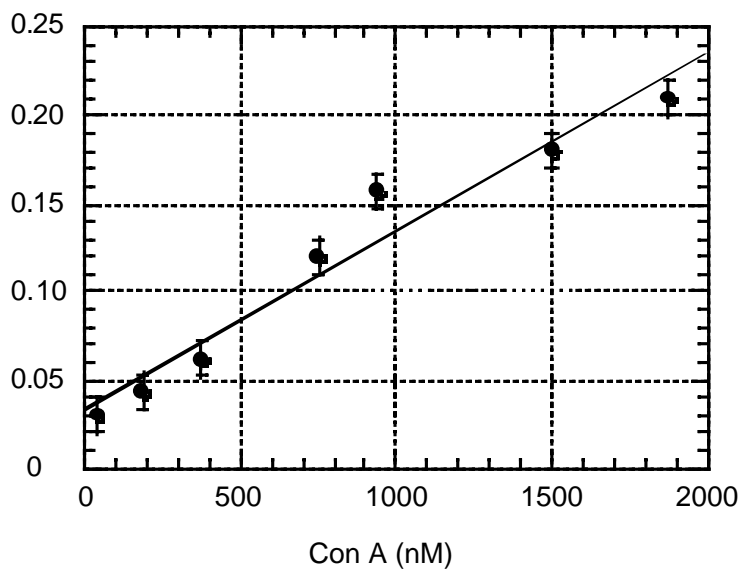

Figure 4. Magneto binding assay of protein Concanavalin A. The relative magnetic permeability is shown as a function of the concentration of Concanavalin A. In (a) the detection of various concentrations is illustrated, and in (b) the focus is on the linear range of the response.

\section{Visions of the future}

The present development of analytical equipment is heading towards smaller and more compact instrumentation. An increased need for patient monitoring, industrial quality control and environmental survey creates a demand for portable and rugged equipment, which can perform accurate and sensitive laboratory and in-field measurements. Considering these facts, we strongly believe that biomagnetic transducers applied in biosensors and bioassays are providing the endusers with a new and promising technology. Further attractive features of this technology include simple approach, rapid results, multi-analyte detection, low-cost per measurement, stable and non-hazardous reagents, and reduced waste handling. Thus, we foresee that it is only a question of time before the magneto-based bioanalysis will become a serious competitor to the established analytical methods.

\section{References}

1. Heineman, W.R.; Halsall, H.B. Anal. Chem. 1985, 57, 1321A.

2. Janata, J. J. Am. Chem. Soc. 1975, 97, 2914.

3. Clark, L.C. Jr; Lyons, C. Ann. NY Acad. Sci. 1962, 102, 29.

4. Sethi, R.S. Biosens. Bioelectron. 1994, 9, 243-264.

5. Mandenius, C.F.; Mosbach, K.; Welin, S.; Lundström, I. Anal. Biochem. 1986, 157, 283.

6. Aizawa, M. Denki Kagaku 1982, 50, 981.

7. Kronkvist, K.; Lövgren, U.; Edholm, L.-E.; Johansson, G. J. Pharm. Biomed. Anal.1993, 11, 459.

8. Schultz, J.S.; Sims, G. Biotechnol. Bioeng. Symp. 1979, 9, 65.

9. Scheller, F.; Schubert, F. Techniques and Instrumentation in Analytical Chemistry; Biosensors, Elsevier, The Netherlands, 1992, Vol 11.

10. Wolfbeis, O.S. GBF Monographs 1987, 10, 197.

11. Mattiasson, B.; Danielsson, B. Carbohydr. Res. 1982, 102, 273.

12. Danielsson, B.; Mosbach, K. Methods Enzymol. 1988, 137D, 186.

13. Yalow, R.S.; Berson, S.A. Nature 1959, 184, 1648-1649.

14. Tijssen, P. In: Practice and Theory of Enzyme Immunoassays (Burdon RH, van Knippenberg, PH, eds) Elsevier, Amsterdam, 1985.

15. Oellerich, M. Journ. Clin. Chem. Clin. Biochem. 1980, 18, 197-208.

16. Anderson, D.J.; Guo, B.; Xu, Y.; Ng L.M.; Kricka, L.J.; Skogerboe, K.J.; Hage D.S.; Schoeff, L.; Wang, J.; Sokoll, L.J.; Chan, D.W.; Ward, K.M.; Davis, K.A. Anal. Chem. 1997, 69, 165R-229R.

17. Shons, A., Dorman, F.; Najarian, J. J. Biomed. Mater. Res. 1972, 6, 565.

18. Guilbault, G.G.; Ngeh-Ngwainbi, J. GBF Monography 1987, $10,187$.

19. Malmqvist, M. Portland Press Proc. 1993, 4, 61.

20. Flanagan, M.T.; Pantell, R.H. Electronics Lett. 1984, 20, 968.

21. Schultz, J.S.; Mansouri, S.; Goldstein, I.J. Diabetes Care 1982, 5, 245.

22. Kriz, D.; Kriz, C.B. US patent application 08/847,125 (priority date August 20,1995).

23. Pieters, B.R.; Williams, R.A.; Webb, C. In: Colloid and Surface Engineering (Williams, RA, eds) ButterworthHeinemann, Oxford, 1992, 249.

24. Molday, R.S.; Yen, S.P.S.; Rembaum, A. Nature 1977, 268, 437-438.

25. Kronick, P.L.; Campbell; G.; Joseph, K. Science 1978, 200, 1074-1076.

26. Hersh, L.S.; Yaverbaum, S. Clin. Chim. Acta, 1975, 63, 69-72.

27. Nye, L.; Forrest, G.C.; Greenwood, H.; Gardner, J.S.; Jay, R.; Roberts, J.R.; Landon, J. Clin. Chim. Acta 1976, 69, 387-396.

28. Kriz, C.B.; Rådevik, K.; Kriz, D. Anal Chem 1996, 68, 19661970.

29. Kötitz, R.; Matz, L.; Trahms, H.; Koch, H. IEEE Trans. Appl. Supercond. 1997, 7, 3678-3681.

30. Babb, C.W.; Coon, D.R.; Rechnitz, G.A. Anal. Chem. 1995, 67, 763-769.

31. Baselt, D.R.; Lee, G.U.; Colton, R.O.J.; J. Vac. Sci. Technol. $B$ 1996, 14 (2), 789-793.

32. Baselt, D.R.; Lee, G.U.; Hansen, K.M.; Chrisey, L.A.; Colton, R.J. Proc. IEEE 1997, 85, 672-680.

33. Baselt, D.R.; Lee, G.U.; Natesan, M.; Metzger, S.W.; Sheehan, P.E.; Colton, R.J. Biosens. Bioelectron. 1998, 7-8, 731-739.

34. Kriz, D.; Kriz, C.B. Swedish patent application SE 9502902$1,1995$.

35. Kriz, K.; Gehrke, J.; Kriz, D. Biosens. Bioelec. 1998, 13, 817823. 